\title{
Human, Organisational and Societal Factors in Robotic Rail Infrastructure Maintenance
}

\author{
David Golightly ${ }^{1, *}$, Jamie Chan-Pensley ${ }^{2}$, Nastaran Dadashi ${ }^{3}$, Shyma Jundi ${ }^{2}$, Brendan Ryan ${ }^{3}$ and Amanda Hall ${ }^{4}$ \\ 1 School of Engineering, Stephenson Building, Newcastle University, Newcastle NE1 7RU, UK \\ 2 Connected Places Catapult, The Pinnacle, 170 Midsummer Blvd, Milton Keynes MK9 1BP, UK; \\ jamie.chan-pensley@cp.catapult.org.uk (J.C.-P.); shyma.jundi@cp.catapult.org.uk (S.J.) \\ 3 Faculty of Engineering, University of Nottingham, Nottingham NG7 2RD, UK; \\ nastaran.dadashi@nottingham.ac.uk (N.D.); Brendan.Ryan@nottingham.ac.uk (B.R.) \\ 4 Network Rail, The Quadrant:MK, Elder Gate, Milton Keynes MK9 1EN, UK; amanda.hall@networkrail.co.uk \\ * Correspondence: david.golightly@newcastle.ac.uk; Tel.: +44-(0)-0191-208-6000
}

check for updates

Citation: Golightly, D.; Chan-Pensley, J.; Dadashi, N.; Jundi, S.; Ryan, B.; Hall, A. Human, Organisational and Societal Factors in Robotic Rail Infrastructure Maintenance. Sustainability 2022, 14, 2123. https:// doi.org/10.3390/su14042123

Academic Editor: Marco Guerrieri

Received: 6 January 2022

Accepted: 10 February 2022

Published: 13 February 2022

Publisher's Note: MDPI stays neutral with regard to jurisdictional claims in published maps and institutional affiliations.

Copyright: (c) 2022 by the authors. Licensee MDPI, Basel, Switzerland. This article is an open access article distributed under the terms and conditions of the Creative Commons Attribution (CC BY) license (https:// creativecommons.org/licenses/by/ $4.0 /)$.

\begin{abstract}
Robotics are set to play a significant role in the maintenance of rail infrastructure. However, the introduction of robotics in this environment requires new ways of working for individuals, teams and organisations and needs to reflect societal attitudes if it is to achieve sustainable goals. The following paper presents a qualitative analysis of interviews with 25 experts from rail and robotics to outline the human and organisational issues of robotics in the rail infrastructure environment. Themes were structured around user, team, organisational and societal issues. While the results point to many of the expected issues of robotics (trust, acceptance, business change), a number of issues were identified that were specific to rail. Examples include the importance of considering the whole maintenance task lifecycle, conceptualizing robotic teamworking within the structures of rail maintenance worksites, the complex upstream (robotics suppliers) and downstream (thirdparty maintenance contractors) supply chain implications of robotic deployment and the public acceptance of robotics in an environment that often comes into direct contact with passenger and people around the railways. Recommendations are made in the paper for successful, human-centric rail robotics deployment.
\end{abstract}

Keywords: robotics; human factors; railway; maintenance; organisational change

\section{Introduction}

Rail infrastructure requires maintenance to ensure continuous availability and safe performance. Track, overhead line equipment and structures such as bridges, tunnels and embankments require routine inspection. Detected faults and predictions of potential failure then trigger maintenance activity ranging from a simple, on-the-spot remedial action to prolonged infrastructure reconstruction with substantial disruption.

Much of the work, both inspection and maintenance, still relies on human effort. This work occurs in a potentially hazardous environment, where track workers need protection from nearby service or engineering trains [1,2]. It can take place in remote or difficult to access locations such as tunnels, at night and in poor weather [3-5] and requires complex communications that are prone to error [6]. It may also require physically demanding work such as the manual handling and mounting of catenary equipment $[7,8]$. Inspection and maintenance require consistent, time-intensive, high-quality work from a skilled but ageing workforce [9] and complex decision making with a burgeoning volume of asset data [10].

There is therefore a growing need to apply autonomous maintenance and inspection technologies to reduce physical exposure to hazards, improve the volume and speed of data collection and analysis and offer the opportunity to release human expertise from physical labour for problem solving and decision making. Potential autonomous technologies involve robotics that support the inspection and/or maintenance of track assets, such as 
tunnels [11-14] and rail-mounted autonomous vehicles or unmanned aerial vehicles (UAVs) to support inspection and maintenance $[15,16]$.

The introduction of robotics has a number of sustainability aspects. First, rail transport is a low-carbon form of transportation for both passengers and freight [17]. The continuous availability of the rail network, through high-quality maintenance, is essential for the appeal of rail as a transport mode. Second, the viability of the rail service is also essential to the long-term financial sustainability of rail transport, ensuring its competitiveness against other modes such as road freight, private car and short-haul aviation [18]. Finally, the safety of workers, and the ability for them to work healthily and productively to a longer age, is a key aspect of the social sustainability of the railways.

While the technical innovation around these forms of inspection and maintenance is developing at pace, far less is known about the human and organisational challenges associated with such technology. Evidence from the adoption of other forms of intelligent maintenance technology, such as predictive maintenance tools, is that work requires new skills, working practices and organisational configurations $[19,20]$. Similarly, the adoption of robotics in areas such as manufacturing suggests the need for a user-centred approach to the design and deployment if there is to be both satisfactory performance and buy-in from the workforce [21,22]. Finally, the rail sector needs to consider public perceptions of autonomous technologies [23], both as a major organisation with high public visibility and also because of interfaces with the public when people live near the railway or interact at places such as level crossings [24]. The arising human-factors-related research questions are therefore:

(1) What are the user-centred design and acceptance issues that face the introduction of autonomous rail maintenance systems, in terms of individual, organisational and societal concerns?

(2) How can our understanding of these issues be used to identify effective means for working with users (i.e., rail staff) and the public to get the maximum benefit for them and for the railways as a whole? The following paper addresses these questions through use of a scenario as the basis for 25 semi-structured interviews with experts both from rail and from other domains with experience in robotics and UAVs. The analysis of these interviews identifies a structured set of factors that need to be anticipated for successful adoption. Additionally, the interviews highlight frameworks from existing human factors work - both from rail and from other domains-that can be applied, with adaptation, to support user-centred autonomous maintenance. These contributions point towards future design, deployment and engagement strategies for human-centred adoption of autonomous rail maintenance.

\section{Background}

One of the major barriers to the successful deployment of technology is that development or deployment tends to be viewed purely in technical terms. In short, the technology is developed purely with a view to meeting technical or functional needs, and then users of the technology are required to learn how to use it and adapt around it. This presents several challenges:

- There is suspicion or outright rejection of the technology [25].

- The technology requires significant training and/or users do not have a good understanding of how it works, leading to a negative impact on performance [26].

- The technology changes work in unanticipated ways, which may have unanticipated reverberations across working practice [27].

- The technology provides an initial advantage in reducing workload, but, over time, mechanisms (particularly automated mechanisms that were intended to assist work) drive new expectations of performance and capacity or new levels of reliance on technology that was only originally intended to assist [28,29].

When technology is designed and deployed appropriately (with a view to how people will use technology and where potential errors or failures can be anticipated), the results 
are a technology that can be adopted rapidly and effectively to deliver high-performance working, reduced development, training and operational costs and acceptance and positive adoption by intended users [30].

It is therefore essential to consider the range of factors that might influence the successful design and deployment of technology. Key to this is the 'socio-technical systems' view, where technology, people, processes (both formal and informal) and organisational structures (e.g., teams, both formal and informal) combine to create work performance [31-33]. In addition, the ethics and public perception of robotics also introduce a societal aspect to considering the human implications of widespread robotic use.

In a similar context, ref. [34] applied a socio-technical framework, based around that of Wilson and Sharples [32], to understand the deployment implications around remote detection systems for railway infrastructure-capturing issues at user, workplace and organisational levels. In terms of industrial robotics, ref. [35] reviewed factors influencing workers' acceptance of human-robot collaboration at work and suggested that aspects associated with the robot (object-related), worker's background (subject-related) as well as organisational environment (context-related) will all influence workers' acceptance.

Some potential examples of issues of rail robotics at these different levels are discussed below.

\subsection{Users}

There are a number of known implications for the design of automation systems, including robotics that might impinge on those directly working with the technology. Trust in robotics is a major theme [36,37]. Balfe et al. [38] found that trust in automation was directly related to factors such as reliability and competence of automation given a set of tasks, and trust was diminished when the automation was unable to work in combination with humans and to match human expectations. These are primarily qualities relating to transparency of the intentions of the robot, whereas environmental factors play a far smaller role in trust. Reliability levels of different automation have differential effects [39] depending on the cognitive function affected in the user.

All of these issues are as pertinent to robotic systems as they are to other forms of control automation. Ref. [40] emphasised the need to explore trust in the context of humanrobot collaboration prior to its implementation. Ref. [41] conducted a series of experiments on robots from different domains to explore the impact of robot attributes such as form and functions in the human-robot trust relationship. Ref. [42] proposed and tested a scale to evaluate trust with industrial robots, coming up with ten items related to robot's motion, safe co-operation and robot and industrial gripper reliability. One of the key findings was the need for more robust understanding of trust-related constructs, and they presented a list of attributes that shape trust.

Typically, working with another actor (human or robot) requires effort for both parties to maintain a mutual understanding and co-ordination [43]. The design of autonomous technology (robot, UAV or decision-support) and the means it has to communicate with its operator will either optimise or potentially limit that co-ordination, which will impact the associated workload [44]. In addition, workload will increase as a remote operator may be required to control more autonomous agents [45,46]. Scholtz [47] described this as the migration to supervisory interactions with robots, where these interactions are more concerned with having an overview of the situation, mission or task planning, understanding capabilities of the robot(s) and potentially understanding group robot behaviours. At this stage, the autonomous system has become a multi-level control system, which places the requirement less on understanding particular tasks of a given robot and more on understanding the whole performance parameters, goals and capabilities of the agents as a group [48]. 


\subsection{Teamworking}

Rail maintenance work is typically a team activity, involving groups of people taking temporary control of parts of the network, performing the work of bringing materials and plant to the worksite, making repairs and making the area suitable to hand back to the network controller [49]. This work involves wider teamworking and communication to protect the worksite with signals, isolate electrical power supplies and so on [5]. This means that any robot needs to be considered within a task environment that involves multiple people and tasks.

As the situation becomes more complex-as multiple people and/or robots enter the situation-human awareness needs to adapt accordingly. This can be supported by human-machine interface (HMI) and communications between the robot and operator but also by verbal, visual and other cues and knowledge of processes shared between all members of a team, including robots. All of these are shaped by the general 'mission goals' of the team. [50] proposed five categories of interaction awareness in more complex robothuman teams (their focus is around the use of automation in search and rescue scenarios). Understanding of all the potential interactions within the system helps to classify the role and responsibilities to develop situation awareness. These are:

1. Human-robot,

2. Human-human,

3. Robot-human,

4. Robot-robot,

5. The human's overall mission awareness.

In terms of safe working of teams on track (but with implications for broader safety), ref. [3] discussed forty main factors that influence safe behaviour and safe culture for rail maintenance teams. The factors that are particularly impacted through the introduction of automation may include:

- Communication: Poor communication between the team members through using inconsistent automated systems (e.g., utilising different legacy systems or different mixes of legacy and new technology at different locations).

- Rule dissemination: Lack of clear instructions and poor implementation of autonomy will potentially lead to misunderstanding rules.

- Competence capability and certification: Automation may lead to reduced competence which will also have an impact on the quality of human intervention in the case of system failures. Appropriate checks and balances should be in place to facilitate successful supervisory control.

- Working hours-different behaviour out of normal hours: Shift work, fatigue and level of vigilance can be modified when automation is more centrally introduced within the workplace.

Furthermore, it is necessary to perform an analysis that will encompass all manner of roles involved in the planning of maintenance or involved in concurrent activities while maintenance and inspection are taking place (e.g., signaller and electrical control room operators involved in protection and isolation). Ref. [51] provided examples of how to manage this procedural impact change in the deployment of metro predictive maintenance technology, and [52] provided a mapping of rail work environment, with an example of the impact of the introduction of UAVs into rail operations and the impact on human functions.

\subsection{Organisational Factors}

As the automation changes, the roles involved with the automation will fundamentally change, as operators are potentially tasked with operating more autonomous agents and at more remote locations. Ref. [53] noted that despite apparent benefits regarding increased productivity and cost reductions, there are few practical examples of the successful introduction of collaborative robots (cobots). Their comprehensive review listed a number of organisational success factors, including employee-centred factors such as fear of job loss 
and employee acceptance, and they highlighted the importance of good communication with staff as a tool to stronger adoption.

Work in e-maintenance in other sectors $[19,20]$ has already noted the challenges that come from centralising some aspects of maintenance control and monitoring, while there may still be a need for technicians on the ground to implement repairs. Unless an explicit effort is made to support channels of communication and knowledge sharing, silos of working can appear within an organisation, and, in particular, local knowledge and context for the performance of assets (and potential diagnosis of failure) are lost to a central maintenance function.

The introduction of robotics requires new roles and skills [47]. These are more than just the simple repair and upkeep of robots, as it also encompasses understanding robot plans, status of robotic sensors and ongoing management of the performance of the robot, particularly during exceptions. The future robot maintenance role may be highly decentralised, where support and exception handling is supported by expertise provided through cloud-based services [54]. This might place new challenges on the co-ordination of knowledge regarding the performance of maintenance systems, particularly if the maintenance function is provided by a third party.

The introduction of robotics into organisations also requires a cultural shift. A case study of the implementation of robot manufacturing has highlighted a specific range of cultural factors that would influence successful human-robot collaboration [21] which has been applied to rail rolling stock robotic inspection [55]. The critical factors identified are:

- Enablers:

Operator participation in the implementation;

Communication of the change to the workforce;

Visible senior management commitment and support to the project;

Provision of training to the workforce;

Empowerment of the workforce;

Use of a process champion during the implementation.

- Barriers:

Lack of union involvement;

$\bigcirc \quad$ Lack of awareness of the complexity of the manual process by the system integrator;

- Capturing the variability of the manual process prior to introducing the automated system;

Allocation of resources for the development of the automated system.

Another general factor that is critical to the success of any new technology within an organisation is e-readiness [56], which can be captured within the TOE (technology, organisation, environment) framework. A survey of the relevance of TOE considerations for e-maintenance [19] highlighted the critical role of human capital in the successful adoption of e-maintenance.

As well as changes within an organisation, the technical supply chain required to deliver both autonomous systems and to deliver rail maintenance and engineering has a bearing on how well people and teams will be positioned to adopt new forms of autonomous working. Studies of the move from product supply to servitisation in areas such as automotive and aerospace have found a greater need for ongoing communication between service providers and customers [57]. One of the major motivations for this is the need for knowledge sharing. With automation and intelligent technologies being so nascent and, often, very context-specific, there is a need for users of technology to keep in contact with the technical supply chain to ensure that knowledge about the design and operation of technologies is still available. This may involve a contractual agreement between all parties to make sure that knowledge is maintained and continuously available, particularly when asset management and monitoring technology apply to critical assets $[24,58]$. 


\subsection{Societal}

At a public level, different national cultures and cultural norms [59] have been predicted to influence attitudes towards automation. In a study of attitudes to passenger aviation, participants from the USA were less likely to be trusting of automation in comparison to participants from India [60]. This relationship was explained by overall cultural norms of greater collectivism in India which leads to a more trusting and positive attitude in others, in comparison to the individualistic cultural norms of the USA. In addition, uncertainty and risk avoidance are a cultural dimension that is argued to influence automation perception. Cultures that are less avoidant of uncertainty and risk may be more willing to accept automation [60].

Other differences include evidence of greater perceived anthropomorphism in robots and the perception that users maintain a higher level of control in China in comparison to Americans [61]. This work highlights another aspect that needs to be considered in cultural acceptance- that different cultural patterns may emerge depending on whether the person is a passive recipient of the automation, as in the case of autopilots, as opposed to when the person is actively working alongside the automation. In addition, while not a cultural study per se, ref. [62] found that age differences in acceptance of robotics were explained by familiarity with technology rather than simply the age of the user. Finally, cultural attitudes towards uncertainty avoidance may lead to national approaches and policies that manage risk. Therefore technology, rather than being perceived as a risk, is seen as a way to manage and mitigate risk and increase positive perceptions [63]. Another factor is cultural norms in turn taking and the ways emotions should be expressed [64]. This kind of cultural factor has already been acknowledged in the potential application of healthcare robotics. Ref. [65] proposed a model of how different cultural factors play into healthcare robotics that could form a starting point for the culturally aware design of other types of robot.

A three-country study [66] found substantial and consistent differences within the countries in terms of acceptance of different uses of UAVs, with large variation in acceptance depending on whether the task was socially orientated (e.g., search and rescue) versus surveillance (e.g., for traffic offences). The visibility and transparency of governance structures and processes to ensure the ethics of robotics is also important in ensuring the wider public trust [23]. Finally, the railways are different from an environment such as manufacturing in that rail work takes place in a public space. Issues such as trespass and vandalism are already concerns for the railway $[67,68]$, and there are places such as level crossings where the railways and public directly intersect (https: / / www.networkrail.co.uk/ communities/living-by-the-railway/, accessed on 5 January 2022). Therefore, robots on the railways may have to be part of a more general communication and publicity strategy to ensure the confidence, safety and expectations of the public.

\section{Method}

\subsection{Material}

A number of approaches were considered to address the research questions presented in Section 1, including workshops, focus groups and surveys. To make best use of time and resources, the approach taken was to interview a range of subject matter experts with knowledge relevant to the topic of rail robotics.

An initial workshop was held with key project stakeholders from Network Rail to generate (1) scope for the interviews, (2) an example scenario that could be used to guide the interview and (3) a first list of interview participants, based on the stakeholders' network of contacts.

From this workshop, three sets of materials were generated:

- A consent and information sheet, sent to all participants. This allowed all participants to have a clear understanding of the study aims, expectations around their involvement and information about their rights to anonymity and data protection;

- A visual scenario describing how automation could be used (presented in Figure 1) this study covered both short-term and long-term applications of automation to the 
railways. This scenario served to give everyone a common understanding of the automation relevant to the study, to give people from outside of rail engineering an idea of how automation could be used in the future and to ensure that the interviews could focus more on the human and organisational aspects, rather than being misdirected into a discussion of technology implementation;

- A question guide was also prepared to shape the interview and to provide a degree of consistency between the three interviewers involved in the project.

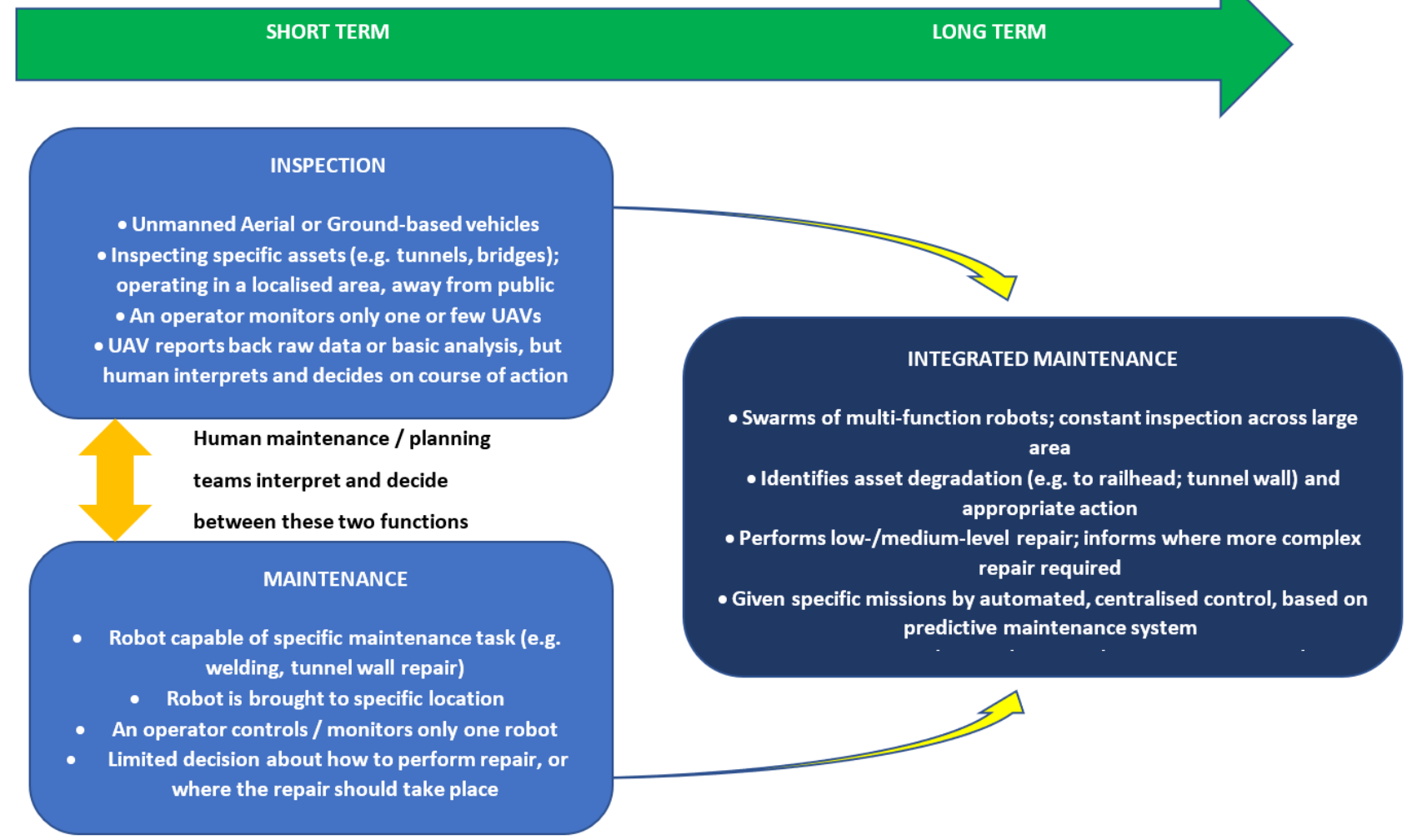

Figure 1. Scenario for discussion in interviews.

Before the start of the study, all materials and procedures were reviewed and approved by the University of Nottingham Faculty of Engineering Ethics Committee.

\subsection{Participants}

Potential participants were identified through contacts of the project team, including those identified at the scoping workshop. Approximately 30-40 experts were approached, with 25 agreeing to take part. Three categories of interviewees were approached:

Rail infrastructure experts-these were a mixture of experts from engineering, asset management and planning functions and those roles associated with training, user-centred design and staff competence. They were included to offer specific insight into the application of automation in rail infrastructure maintenance. In total, there were 12 participants of this category.

Non-infrastructure rail experts-these were experts with rail knowledge but not working in Network Rail. This included industry groups such as Rail Safety and Standards Board (RSSB), members of the supply chain with knowledge of rail engineering safety and processes and academics with knowledge of rail engineering. This category of experts was included in part because they could give perspectives on how automation had been or was being introduced into other aspects of rail. These participants could also offer a wider industry perspective on the benefits and barriers that were likely to be experienced in a rail engineering context. In total, there were 4 participants in this category. 
Non-rail automation experts - these were experts from sectors other than rail who could give their perspective on the factors that lead to successful user-centred use of robotics and other forms of automation. These experts were able to give perspective on a wide range of factors that had led to successful integration of robotics and automation in sectors including military, logistics, automotive and manufacturing. These also represented sectors that, in some cases, were further down the road of automation integration (e.g., manufacturing). In total, there were nine participants of this category.

\subsection{Procedure}

Participants were sent a formal study information and consent letter and an electronic copy of the scenario. They were asked to spend a few minutes in advance of the interview to become familiar with the scenario.

Interviews were most commonly conducted over the phone [69], though, where convenient, some were conducted face to face. The aims of the study were reintroduced, and then the question guide was followed. The approach taken was a semi-structured interview in that participants were given free rein to expand on different topics as they saw fit. This was important as different participants had different expertise relevant to different aspects of the automation framework and were therefore allowed to discuss at length those points where they were most comfortable or had more to contribute.

Each interview typically lasted $45 \mathrm{~min}$. In all cases, extensive contemporaneous notes were taken, particularly for 5 of the interviews where it was not possible to use audio recording due to the interview taking place in a public location. For all other interviews, audio recordings were made and transcribed.

\subsection{Analysis}

Following [70], coding and analysis went through a number of iterative stages. Transcripts and notes were read, and audio was reviewed for each participant. A spreadsheet was developed with a row for each participant record. As well as capturing anonymised participant details (e.g., background, organisation, gender), interview content for each participant was analysed and added to the spreadsheet for:

- Short-term scenario:

User factors;

Team factors;

Organisational factors;

Societal factors.

- Long-term scenario:

User factors;

Team factors;

Organisational factors;

Societal factors.

- Any other comments of note.

Data for each column were then reviewed to identify specific themes within the four levels of factors. These themes were then presented to members of the project team for comment. These comments were then converted into a concept map for each high-level area with a narrative describing themes and sub-themes (similar to the results section below). These outputs were presented in a report that was circulated to all participants including the initial workshop attendees for comment or clarification. This generated a few minor comments that were integrated into the final theme descriptions presented here.

\section{Results}

\subsection{User Factors} below.

Factors pertaining to the user are presented in a concept map in Figure 2 and explained 


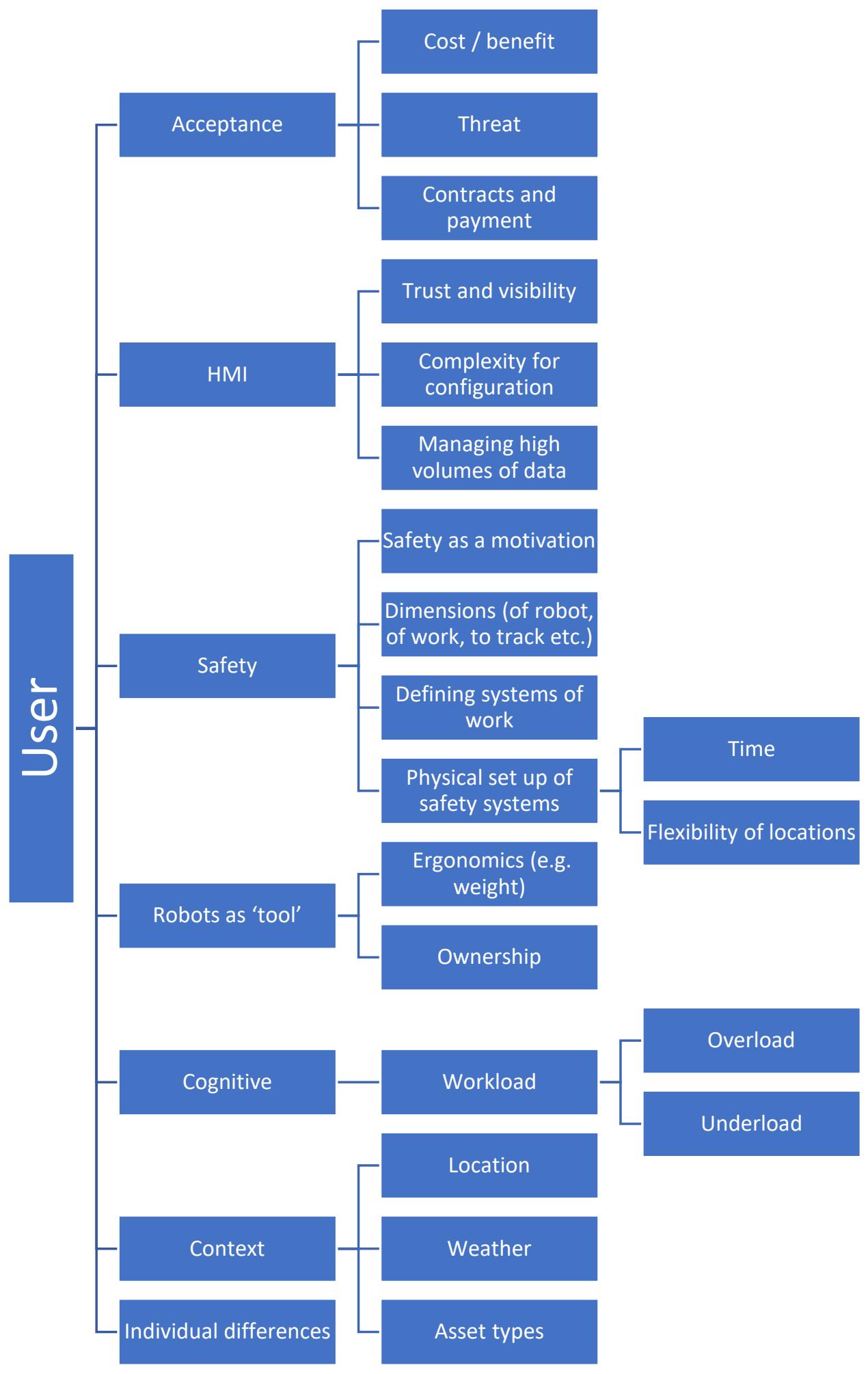

Figure 2. Thematic map for user factors.

\subsubsection{Acceptance}

Acceptance in terms of users covers those factors that will encourage or discourage the adoption of automated systems for maintenance. Automation must be able to do the job better and, primarily, more quickly than the existing manual process. Other benefits were seen in terms of less physical effort or exposure to unpleasant conditions (e.g., working in adverse weather). Benefits and costs apply not only to the maintenance task itself but 
getting on track, execution of the work, getting the equipment off track, etc. Safety would not have to be compromised at any point.

Several experts from within rail put forward the view that staff knew there was a role for automation. The safety, health and viability of the railways as an enterprise was a primary concern, and automation had a role to play in this. However, there was a risk of perceptions of automation as a negative force on employment. Factors to reduce that threat would be a commitment that automation would lead to upskilling ('something you would want to put on your $\left(\mathrm{CV}^{\prime}\right.$ ), that automation was seen to replace only low-value, fatiguing tasks leaving staff to focus on high-value tasks and that there was genuine visibility of resources being redeployed onto useful, high-priority work.

One of the barriers to acceptance would be the impacts on contracts and payment. Staff paid by the hour might be reluctant to use automation if it allowed them to get the job finished earlier. In addition, staff paid by shift might be reluctant to use a technology if that meant they finished quickly and had a lot of spare time (e.g., at night) on their hands.

\subsubsection{Human-Machine Interface}

In terms of robot human-machine interface (HMI), the notion of trust had a number of impacts. First, the automation has to be reliable, and the appropriate task can be consistently performed to the appropriate standard. For automation on track, this becomes a complex problem because the requirements of the task (and therefore the ability of the automation to perform the task reliably) vary greatly due to different conditions, location, asset type, etc.

For the short-term at least, many forms of robots (a) are perceived as niche products that require a high degree of configuration, (b) require specialist knowledge to set up and operate for any location and (c) may come from multiple suppliers, where the HMI of configuration may be inconsistent between suppliers. The result is that programming automation can be complex and require very specific skills often with unforgiving user interfaces. A high degree of technical knowledge on the part of the user should be anticipated with opportunities for upskilling on the part of the operator.

For inspection systems, there were concerns that systems can generate copious amounts of data regarding the asset they are looking at. Representations of the data must be userfocussed if meaningful action and interpretation are to take place. This will involve careful, user-centred HMI design.

\subsubsection{Safety}

Safety was routinely cited by rail experts as the most important motivation for the adoption of autonomous maintenance systems. The potential to remove staff from around the railway or from difficult-to-access locations and maintain structures such as tunnels and bridges would be a major reason to adopt automation. In this regard, the railways could learn specifically from application domains such as nuclear or offshore inspection, where robots play a key role in reducing the human exposure to harm.

As well as the immediate reduction in risk, much trackwork takes place at remote locations (which can require a long drive) at night, where there may be few welfare facilities, and the requirement for shift work. Autonomous robotics could radically reduce the need for this kind of working.

In contrast to 'safety as a motivation', being able to work safely within and around robots emerged as a vital consideration. Safety was more than one single 'entity' and took on a number of directions, each of which was important and needed explicit consideration in design and deployment.

\section{- Safe operation of the automation}

Where the robot is able to operate without causing harm to people in the vicinity. Safe performance of the automation that ensures the safe maintenance of the railway. Behaviour in unsafe situations:

Even though the automation may not be at fault, it needs to be able to respond to that situation in a manner that protects itself and others on the railways. 
Following on from above, new forms of automated working might require the physical set-up of specific safety systems. For current manufacturing systems, for example, configurations of light sensors and tracking are used to hard- or soft-stop the robotics as humans approach. However, setting up similar arrangements at trackside would take time and would further add to the cost of work. One point of discussion was the appropriate role to be responsible for these tasks.

\subsubsection{Robots as 'Tools'}

The practical ergonomics for physical tool design for outdoor equipment should not be overlooked — can it be lifted, carried, accessed for maintenance, etc.?

Also related to the idea of automation as a tool is the notion of ownership. Workers, particularly craft workers in rail such as welders, were known to customise their tools and build a form of attachment with them. Staff, therefore, may want a one-to-one attachment with a specific robot or tool. It might be more cost-effective for staff to 'hot desk' robots, though this would lose the personal and customised element of the tool.

\subsubsection{Cognitive Factors for Working with Automation}

A primary concern was underload. In terms of controlling automation, this might mean at a cognitive level people are struggling to remain engaged with the task. However, in a trackside environment, underload was seen as a more fundamental issue of boredom, keeping warm and even staying awake if previously physical trackside jobs are now stationary monitoring jobs, potentially at night and in the cold. This is likely to be more of an issue where operators are still required to be trackside (e.g., for short-term inspection scenarios).

There are also, however, conditions of high workload associated with automation, particularly in the long-term scenario, where an operator is monitoring multiple forms of automation (e.g., managing the flight of multiple UAVs) or at challenging parts of robotics maintenance such as critical welds or safely moving around complex worksites.

\subsubsection{Context}

Context comprises the specific environmental factors that have contributed to trackwork being perceived as more complex than other working environments such as the factory and was a recurrent theme. Context encompasses:

- Location-understanding general geographic location; the specific location in terms of its proximity to other assets; access to the location; whether the location was isolated or near to urban environments; interfaces with the public (e.g., level crossings).

- Working arrangements - whether the work was being conducted without protection (highly unlikely in the short term but possible in a future scenario where robots had knowledge of the real-time timetable); as a single worksite within a possession or as one of a number of worksites within a possession.

- Physical environment-whether this took place in the day, at night, in rail, cold, sunshine, etc.

- Asset context - what kind of asset was being inspected, variability among assets of the same type; age of the asset, etc.

Being able to adapt to the variability of these different factors was considered vital not only to the viability of automation to perform the required work but also to the understanding of factors such as safety arrangements or the degree of configuration of the automation and therefore knowledge and skill on the part of the operator to programme that configuration or understand its limits.

\subsubsection{Individual Differences}

While context is a source of variation in the operation of rail automation, the operators themselves are a source of variation. Factors that emerged were:

- Operators' different skill levels and their ability to understand and use equipment involving a high degree of technology. 
- Operators' experience, particularly in more 'craft' jobs (i.e., those jobs that might be performed by hand and/or involve a high degree of tacit, physical and sensory knowledge or skill).

- Decision-making ability—not all people had the same ability to engage and make critical decisions.

- Motivations to work - at the very frontline, some of the people attracted to the job were attracted because of the autonomy, working outdoors, physical work, not being compelled to use IT, etc. Therefore, the introduction of robotics could run contrary to what they wanted from the job.

These factors would influence both willingness and ability to work with automation and therefore need careful consideration in competence, training and culture change.

\subsection{Team Factors} below.

Factors pertaining to the user are presented in a concept map in Figure 3 and explained

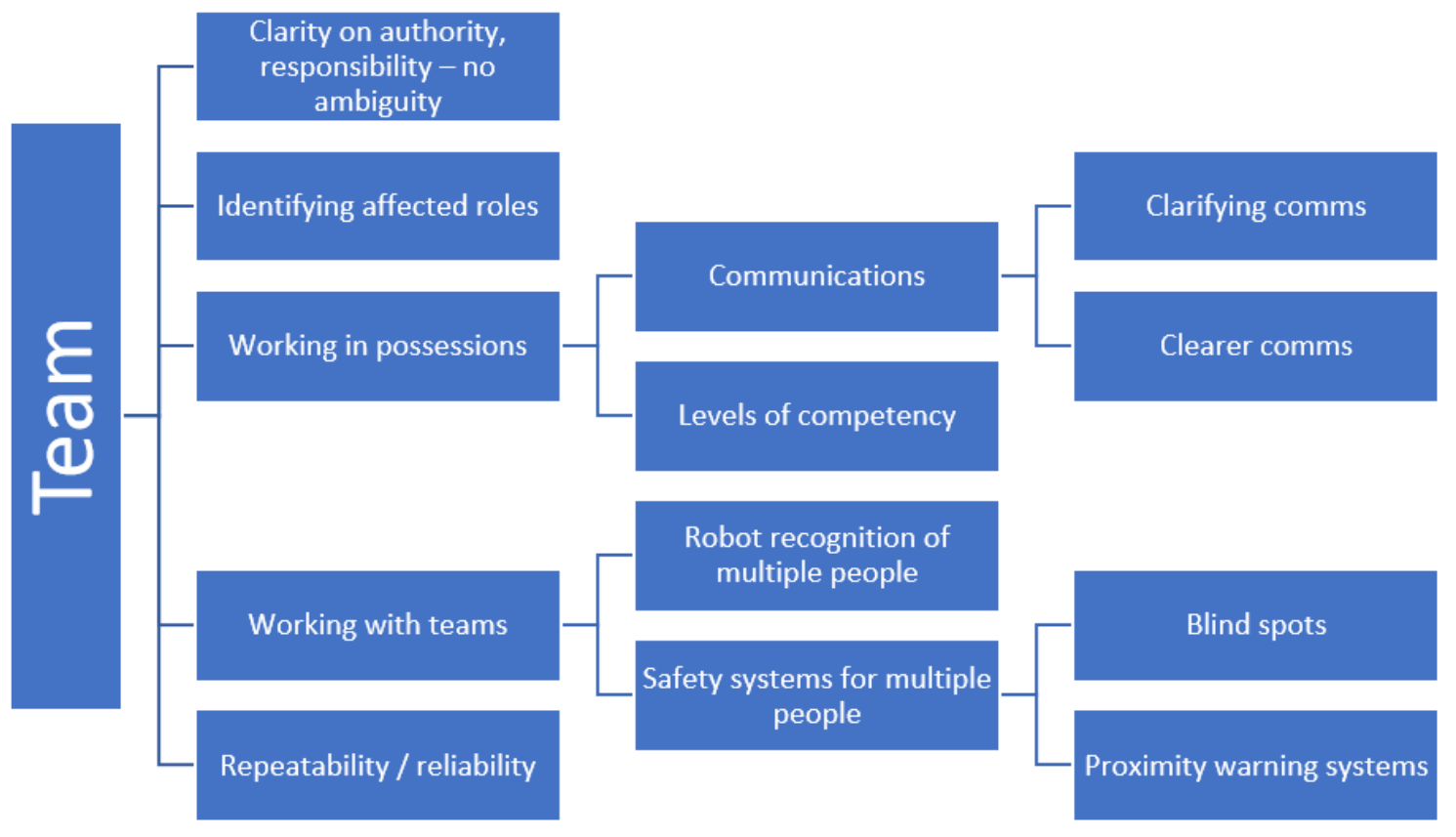

Figure 3. Thematic map for team factors.

While teams have a relevance to inspection, they were mostly discussed in the context of robotics and maintenance with relevance to trackwork within possessions. These are times when areas of the rail network are blocked from normal service trains, often at night or at weekends. These possessions involve complex arrangements of signals set to red to protect workers, the placement of physical barriers to stop the unprotected movement of trains and complex coordination of communications. In order to make best use of time, a single possession may cover several miles and may include separate designated 'worksites' each with their own safety representatives (for more, see $[4,5,49,52]$ ).

\subsubsection{Authority}

Within a team setting, it will need to be clear what the set of authorities and responsibilities are around the automation and to adapt current safe systems of work to reflect the inclusion of a robot. There will need to be clarity on who is responsible for any on-track automation within a team, with an acknowledgment that not all people have the technical skill to operate a robot, and not all have the decision-making skills to act with authority regarding a robot. The lines of authority and responsibility also extend to factors such as 
who is responsible for bringing a robot to and from the site and who is responsible for maintaining the robot.

\subsubsection{Identifying Affected Roles}

The descriptions of automation highlighted the way in which roles will need to change and how many roles will be affected by the introduction of automation. These were across a number of areas:

- Immediate team and trackwork roles-not only those who would be working with or around automation but those who might come into indirect contact with robotics, particularly in larger worksites. It was noted that many of these people may be from the maintenance supply chain.

- Wider asset management roles who would be required to plan or interpret asset maintenance and renewal and therefore use the information that autonomous maintenance systems might capture.

- Wider planning roles who would need to factor in the delivery and management of robots and other forms of automation within their maintenance planning.

- Signalling and controllers of the electrical supply who could be required to provide protection and would potentially play a role in managing the situation should a fault develop (e.g., a robot fails leading to the work overrunning into the expected handback of the network).

- Drivers that need to know what to expect and potential risks if work involving robots is taking place on and around the railway.

A complete review of rules would be required, and this would be enabled by system models of human functions on the railways. In addition, new roles, such as those to maintain stable robots, will be required and are both an opportunity and require appropriate job design.

\subsubsection{Working in Possessions}

A specific area that was raised as a consideration was the ability of a robot to move within a worksite, either independently or with the control of a machine operator or similar role. Particularly in a medium-term scenario where a robot might be moving autonomously within a worksite, the robot would need to give and receive communications in a manner commensurate with safe working within a worksite. This might, for example, involve communicating to other teams as it passes through different worksites within a possession. On the other hand, the ability of the robot to offer clear and unambiguous commands and to respond either unambiguously or to request clarification were all reasons that communications involving autonomous systems may be more effective.

Across this is the need to understand and anticipate varying levels of competency in general and in particular with regards to working with automation that might influence the success of working with robots. This is likely to be affected by people coming in from third-party maintainers or construction companies (see Section 4.3).

\subsubsection{Working with Teams}

Functionally, rail experts highlighted that robots will need certain capabilities to enable them to work effectively with teams in a trackwork situation. For safety reasons, the robots will need to be able to recognise multiple people as there may be more than one person around an area of work, even if they are not directly concerned with controlling the robot. Systems for sensing people will need to be sensitive to blind spots potentially caused by a plant, infrastructure or other people. In addition, proximity warning systems will need to be designed to take into account teamworking, particularly as for many tasks in railway maintenance there is a need for people to get in and around the point of work (e.g., to position equipment or assets). 


\subsubsection{Repeatability/Reliability}

If the same equipment is to be used in different places (e.g., for inspection in multiple sites), there is a need to make sure the results of the inspection or maintenance activities are equivalent and comparable across multiple sites. Outputs may not be identical reflecting the contextual variation between sites, but they should be comparable allowing trust that this is a reliable process and to allow potential data analysis across sites.

\subsection{Organisational Factors}

Organisational factors are presented in a concept map in Figure 4 and explained below. Organisational factors could be subdivided into two groups-within-organisation factors within the infrastructure owner/operator organisation and inter-organisational factors linked to the supply chain. The supply chain was then considered as both an upstream supply chain, providing robots, automation, etc., and a downstream supply chain of contractors providing maintenance work for the infrastructure manager.

\subsubsection{Within}

Leadership

An important organisational enabler of change was the role of senior management to give long-term support and commitment to the automation concept as a whole. This involved a visible commitment to the automation and prioritisation of the project. Within that prioritisation comes an understanding of how the impact of automation relates to other priorities within maintenance such as short-term delivery, costs and safety. The perception was that there would need to be a senior person with responsibility to champion and support the introduction of an autonomous system or systems as a strategic project if it was to have the long-term benefits and outcomes described in the long-term scenario.

\section{Engagement}

Engagement with staff at all levels of the organisation is vital. Experts from within rail cited engagement as being the factor that had ensured the success of effective projects and the lack of engagement as a major factor in those projects that had failed. This is related not just to maintenance but also to several examples of procedural change, technology change, automation implementation in driving or traffic control, etc. Participants from outside the rail sector also cited successful projects as being those who engaged and actively sought involvement from stakeholders. Engagement has twin benefits of giving ownership of the project to its users, thus encouraging their adoption and eliciting their knowledge on the best ways to apply the technology, thus reducing project risk.

Rail experts defined multiple sets of stakeholders, each of which may need to be approached in a slightly different way:

- Decision makers and managers, e.g., track maintenance engineers-need to be convinced of the benefits of the project and technology. How can it improve their work processes, and how can they assess where it is going to have a genuine positive impact? Will it impact on the staff and resources they have available?

- Unions-should be involved to understand the safety and performance benefits, while discussing the potential changes in resourcing that may result.

- Frontline staff- to understand the benefits and to understand their perceptions and gather their expertise on how best to introduce the technology into the working environment and what procedural and training changes will be required.

\section{Phased Introduction}

The introduction of new technology should be phased for a number of benefits. Firstly, it allows the technology to be tested and proven before wider rollout. Secondly, it makes it easier to direct the cultural change, not only because the scope is more managed but also because there are hopefully positive messages to communicate. Thirdly, because of the 
variety of the railway contexts, a single approach is unlikely to work in all places at all times. A phased approach would allow steady adoption and adaptation across different settings.
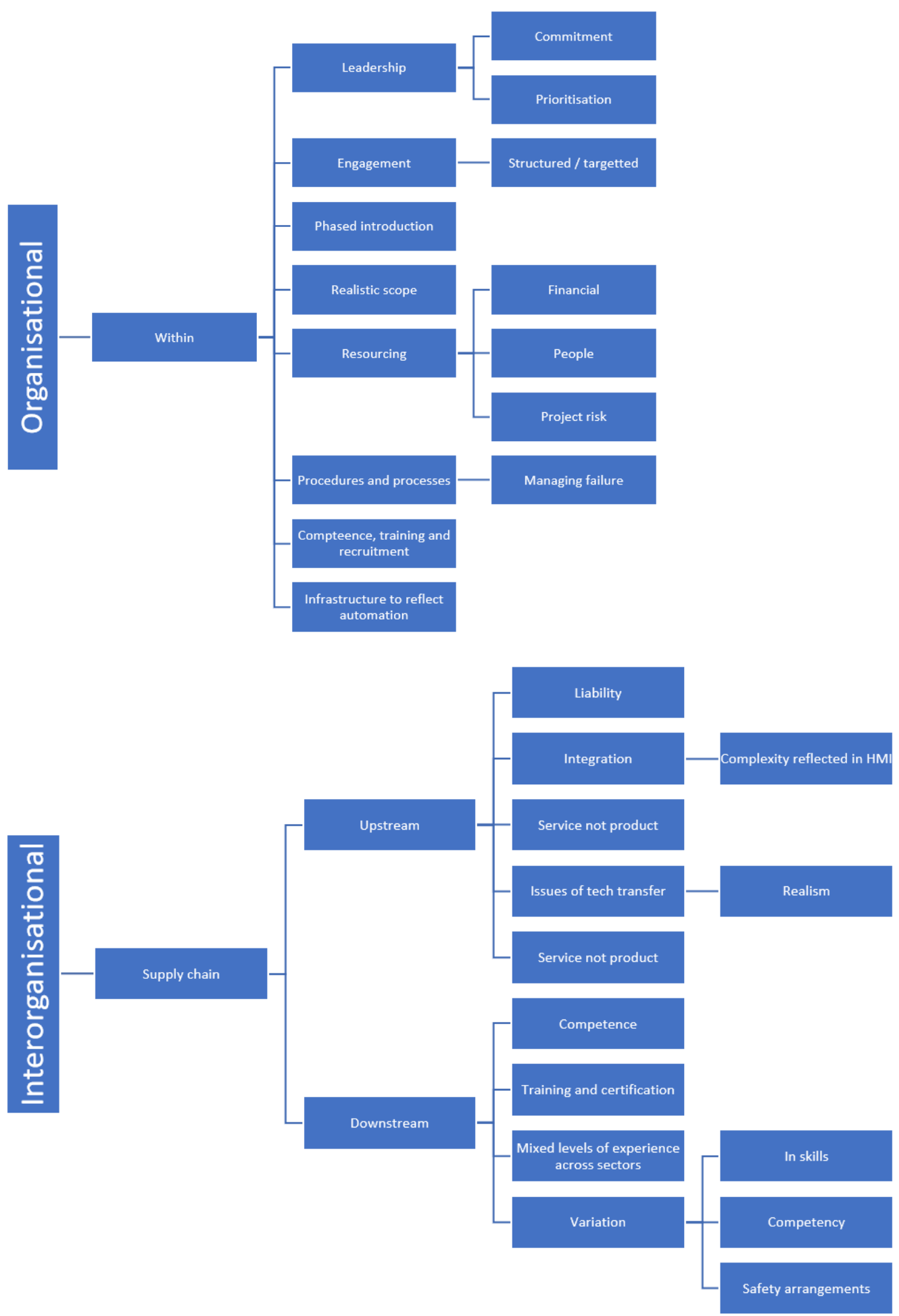

Figure 4. Thematic map for organisational and inter-organisational factors.

One factor that is required to support a phased approach is good knowledge management. There needs to be a way of communicating the benefits and learnings from different sites and implementations to different parts of the organisation. Failure to do so 
in the past has hampered the introduction of technology to support maintenance such as semi-autonomous welding equipment.

\section{Realistic Scope}

Realism needs to be applied when introducing new technologies, and this will apply to autonomous systems for maintenance. Examples were given of projects that had overpromised on what they could deliver but then needed substantial customisation to actually work as required. Not only does a realistic scope mean that the technology is more likely to deliver the intended benefits, it helps to build the confidence of operational staff.

\section{Resourcing}

Appropriate resourcing of a robotics project was perceived to take a number of forms:

- Financial resourcing - the kind of projects being described are likely to take time and run over more than the control period. Therefore, as well as there being sufficient financial resources in the short term, there has to be a long-term commitment to fund the project over extended periods.

- People-when projects of this type are initially launched, they tend to take more of people's time, not less, as they learn to use the new process. Often (e.g., for the use of new forms of inspection), they need to run in parallel with the existing processes until they are tested against existing processes and become established. In addition, resourcing should not be reduced because the technology has made efficiency gains but should be redeployed into other high-priority tasks.

- Appetite for project risk - projects of this type take time and are unlikely to work first time. There will be changes to plans and to scope as the capabilities and limitations of autonomous technology become clear. Projects must be planned and delivered with an understanding that, for the short term at least, there will be risks and problems to solve. This also relates to the culture of the project where people are allowed to fail and to learn without fear of reprimand.

\section{Procedures and Processes}

One issue for both inspection and maintenance is how to embed new technologies within procedures and processes. Experiences from previous rail projects to introduce new maintenance technology had highlighted that the approach taken should not be to have processes for working the automation but to have processes for performing the task with the automation embedded. Similarly, training should not be on how to work the technology per se but how to complete processes and tasks that involve new technology. There is a need to have a clear approach to managing failure, and processes and procedures need to be documented to that effect. While this relates to the major safety failures (e.g., a train colliding with a UAV), it is just as much about the smaller production failures. For example, if a robot fails during some trackwork, what is the process for deciding on whether it is possible to proceed with the work or not?

\section{Competence, Training and Selection}

As discussed under Users (Section 4.1), people are variable both in their pre-existing skill and knowledge and, most likely for automation, in terms of their perceptions and motivations to use the technology. Therefore, a flexible approach to training must be taken. In addition, selection, particularly for those roles that will be in direct control of the automation, is likely to be an issue. While other sectors have found that newer recruits are more likely to be tech-savvy, the perception was that there are still many people coming through to the rail sector who are either under-confident or reluctant to use technology.

\section{Infrastructure to Reflect Automation}

While the short-term scenario is likely to involve existing infrastructure and assets, the kind of long-term benefits of automation may only come about if there is more effort to make 
the infrastructure amenable to automation. In the same way that automotive manufacturing is designing vehicle manufacture so that it is optimised for robotic construction, the railways could adapt infrastructure design to make it more standardised and more amenable to automated construction and maintenance.

\subsubsection{Inter-Organisational-Upstream}

Inter-organisational issues fell into two areas. Upstream issues in the supply chain relate to those organisations supplying hardware and software to support automation. Particularly for new technologies, this might be more than one organisation working together to deliver a trial technology. Downstream inter-organisational issues relate to those when the maintenance or inspection work is being performed by organisations outside the main infrastructure manager. These may be large organisations or smaller suppliers.

Liability

One of the issues raised was liability and chain of responsibility when things go wrong. While this is a contractual issue, it is also a human issue. The chain of responsibility must be known to the operators of equipment so that when something goes wrong, they can find out how to get it fixed. It is also important that frontline operators know that if something goes wrong that has a negative impact on the work (for example, a robotic failure leads to an engineering work overrun), the appropriate person or supplier will be held accountable.

Integration

As noted under HMI, the complexity of the supply chain is reflected in the complexity of the integration of software and hardware. A more complex, temporary supply chain is more likely to make the software difficult to interpret and therefore make faults harder to diagnose. In addition, the role of the integrator (whether in-house or third-party) is to make sense of and implement standards. These are both standards for robots (e.g., ISO 10218) and standards/guidance for how the railways are operated [71]. This interpretation of standards is one of the most challenging tasks for integrators, and complexities and errors will be reflected in either faults in the automation or in the mismatch between automation, HMI and task, which can lead to a lack of trust.

\section{Issues of Technology Transfer}

Many technologies on the railways are adaptations of technologies already developed from other sectors or from existing rail organisations in other countries. For technologies transferred from other sectors, there needs to be an acceptance first of all of the differences in the rail sector (and the variability within that sector) and therefore that (a) technology may need substantial customisation, which will also apply to the training and HMI associated with that technology, and (b) the successes found in other domains may not be found, at least initially, within rail, which highlights the importance of accepting project risk, of providing sufficient resource and of making sure there is a clear and realistic scope for the technology.

\section{Service Not Product}

All of the issues above further introduce project risk. Lessons learned from other sectors, and from other forms of technology introduction in rail, suggest that as a project team (including the rail stakeholder and technology suppliers), it is important to learn about the technology and the requirements it has to meet so that the project changes and knowledge develops. Even when the technology is running and operational, circumstances and staff can change, and knowledge is lost. Therefore, the most effective means to keep a product developing and for knowledge to be maintained is to have a service-type rather than product-type contractual relationship, which lasts over an extended period. 


\subsubsection{Downstream}

Levels of knowledge and training are very variable within this supply chain, as are safety management processes within these organisations, reflecting the wide variety and size of organisations involved [72]. Importantly, not all of these organisations (or their staff) work exclusively in rail. As they come to rail engineering work, they may bring different levels of experience of technology. In some cases, this may be a benefit, for example, if highways maintenance is faster at adopting robotics than rail, contractors who work in both environments can bring their knowledge to the rail sector. Overall, future training and accreditation (e.g., in Great Britain, this is managed under the Rail Industry Supplier Qualification Scheme-RISQS (https: / / www.risqs.org/, accessed on 5 January 2022) for suppliers would need to be adapted to take experience and competence working both with and around robots into account.

\subsection{Societal Factors}

Factors pertaining to society are presented in a concept map in Figure 5 and explained below.

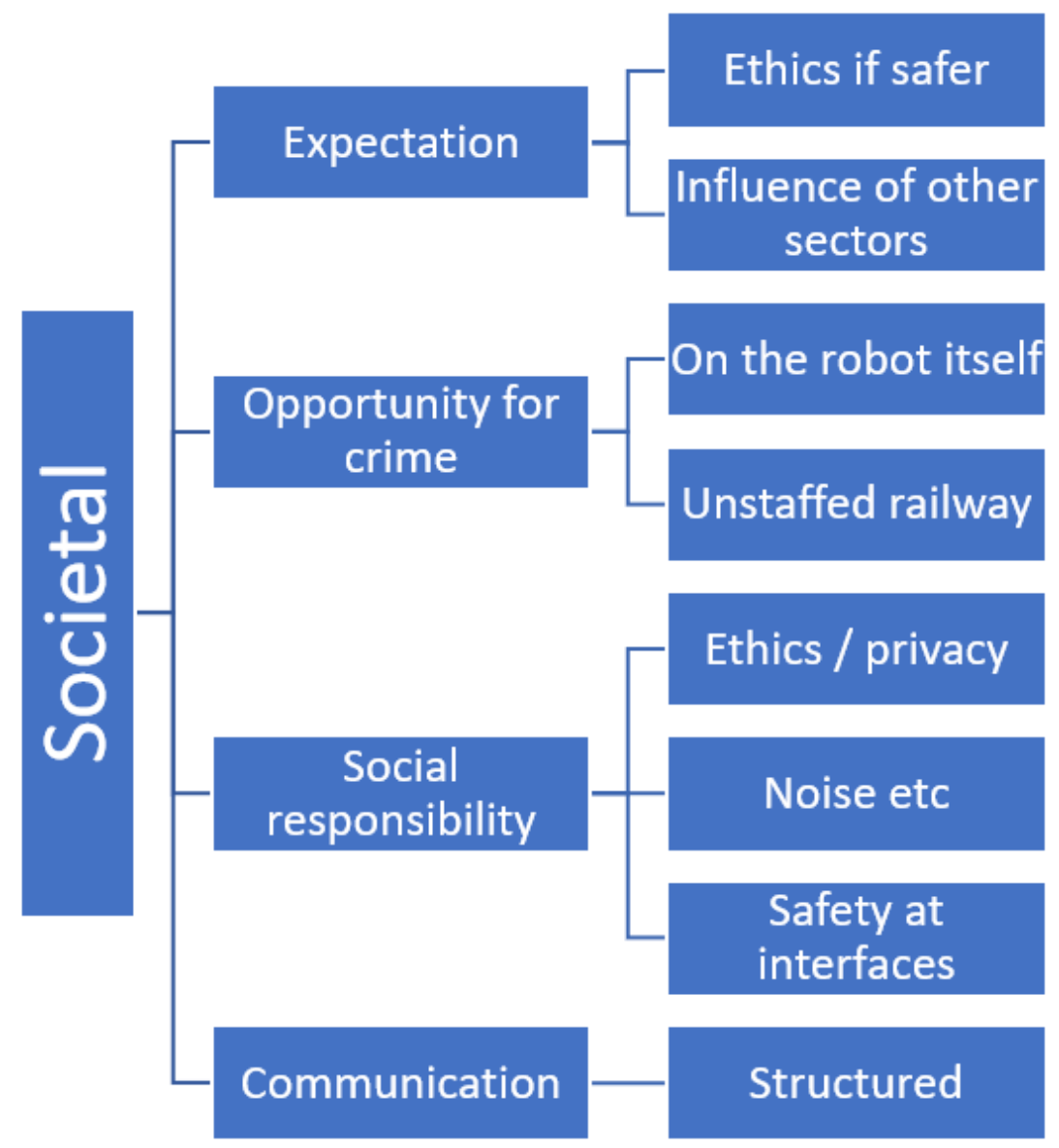

Figure 5. Thematic map of societal factors.

\subsubsection{Expectation}

Rather than experts assuming there will be resistance from society, there was a view that if rail maintenance is safer (both for staff and in terms of the quality of work) and more efficient with the use of automation, there would be an expectation and even an ethical obligation to use robots on the railways. This might become more apparent as robotics and automation are introduced into other maintenance environments such as highways. Moreover, if successful, the rail staff themselves will be advocates for the introduction of robotics and automation as it makes their work safer and more productive. 


\subsubsection{Crime}

One of the perceived drawbacks of the more long-term scenarios for automation was a reduced human presence on the railways. This introduces the possibility of crime. On one hand, this could be criminal damage to the robots and automation itself but also the perception that the railway might be perceived as being increasingly unstaffed and therefore criminal acts around the railways, including those to members of the public at stations, may be more likely.

The counter view is UAVs may provide an opportunity for greater observation around the railway, thus reducing crime; however, this comes with an ethical and social responsibility around privacy.

\subsubsection{Social Responsibility}

The point on crime relates to a broader point of how robotics and automation are viewed formally within a rail infrastructure manager's programme of corporate social responsibility. In the long-term scenario, where these forms of automation are increasingly controlled without an overt human presence, there will be concerns from the public around their own privacy. The management of zones where UAVs can and cannot fly or where robots can or cannot come in close contact with the public will need management. Noise may also be a concern, as robots are used on track and around maintenance work. Finally, there is the general concern from the public around their safety near UAVs or robots. For example, the design of a UAV (labelling and colouring) would need to clearly indicate who it was from and its intentions, potentially with an overtly explicit purpose, to curtail any suspicion. This may also include instructions on how to deal with it (e.g., if a UAV crash-lands in a garden).

\subsubsection{Structured Communication}

All of the above indicates the need for structured communication with the public regarding the more widespread use of automation. The view was that in the short term for UAVs, there would be a visible presence that would have to be managed, but robots would remain specialist technology and not a concern to the public until they have a more prominent and autonomous role as suggested by the long-term scenario.

Communication should be structured so that the most important aspects, including the benefits of automation, are communicated widely through general media. However, the details about specific applications of automation should be localised to the area where they are being applied and time-dependent, in a manner similar to the way Network Rail currently communicates regarding noisy engineering work.

\section{Discussion}

\subsection{Observations}

Overall, the analysis identified many themes that would be expected from pre-existing robotics and automation literature. These include:

- User issues relating to trust, workload and robotic HMI;

- Team issues relating to awareness of the robot around multiple actors and changes to roles;

- Organisational issues relating to competence and having planned engagement and deployment strategies;

- Societal issues related to the wider acceptance of robotics.

However, a number of issues emerged that were specific to the railways. The first of these was the importance of the rail context. Robots and UAVs will be used in a variety of physical settings (urban areas, rural and remote areas) and potentially in a range of conditions (cold, at night). Moreover, the infrastructure to be managed will often vary (types of asset, complexity of asset, age of asset). All of these areas of variability have a profound 
impact on both the configuration of the robot and the ability of the robot to perform the work. Flexible robotics and ease of onsite configuration will be key to acceptance.

Secondly, the viability and cost-effectiveness of using the robot should not be judged just in terms of the task to be automated (e.g., welding) but the whole task lifecycle. This means logistics of taking the robot from a depot, getting it on track, configuring, executing the work and removal. Given the noted challenges and safety issues around trackwork, particularly at the beginning and end of the work [5] and the production risks associated with trackwork [4], these steps should not be ignored in any analysis and must be calculated into both the planning and the cost-benefit analysis when deploying robotics.

Thirdly, while the approach in the study had originally sought to capture 'team' issues, it may be appropriate to think about 'worksite' issues. That is, it is not only the configuration of people but also the configuration of plant, other tools, engineering trains entering a worksite and so on. In terms of people, the nature of the worksite and the roles involved means that there will be people involved directly with the robot but also those who need to understand the functioning and predict the behaviour of the robot, even if they are not directly involved. In addition, site safety roles will have different levels of responsibility, particularly for the set-up of any proximity systems. Visualisation and principles for trackwork such as those proposed by [49] should be updated to assess the impact of robotics on trackwork. Furthermore, rather than 'teams' being the unit of analysis, 'worksite' is probably a better unit of analysis.

Fourthly, the supply chain must be reflected in the analysis of robotic work and in subsequent competence, training and accreditation. While this involves the suppliers of robotics (and integration), in the railway maintenance world, it is also important to consider the role of contractors and third parties involved in maintenance and, particularly, large construction projects with many organisations collaborating together. Within these organisations, some may be involved in controlling or working with robotics, but the challenge comes with other maintenance and engineering staff who may not be directly using the robotics but need the competence and knowledge to work safely around the robotics.

Finally, unlike manufacturing, maintaining and operating the railways is both publicly visible and, in Great Britain at least, part of the national conversation. Public expectations will therefore be high that railway robotics can offer good value and safety and can be seen to be at least keeping pace with other sectors. As importantly, the public may come into direct contact with robots. In this regard, rail robotics must be industrial robots but also be designed in a way that is sensitive to public cultural norms $[59,60]$.

\subsection{Recommendations}

Tables 1-3 present robotics at three levels of maturity-short-, medium- and longterm. Benefits and barriers are summarised for each level of maturity. Each table then presents a number of recommendations for successful adoption at each of these three levels. Recommendations are broken down into two categories. First, functional requirements are the technical requirements of robotics that will be needed to encourage their usability, acceptance and cost-effectiveness for tasks. Second, socio-technical requirements are those requirements of people and organisations, in terms of training, culture change, communication and so on.

Table 1. Benefits, barriers and requirements for near-term adoption of rail infrastructure robotics.

\begin{tabular}{lll}
\hline - & Limited functionality; robot will probably have a specific purpose; \\
- & One-to-one control by operator; \\
Functional description & Inspection likely to be more advanced than maintenance; \\
& Still likely to be embedded within something similar to current process \\
& with equivalent working practices (e.g., standard \\
possession arrangements); & Requires high degree of set-up on the day in terms of safety system; \\
- Requires high degree of configuration.
\end{tabular}


Table 1. Cont.

\begin{tabular}{|c|c|}
\hline Benefits & $\begin{array}{l}\text { - } \quad \text { Safety from less exposure to track environment; } \\
\text { - } \quad \text { Ppskilling; } \\
\text { - } \quad \text { Supportial for improved performance on specific tasks; } \\
\text { - } \quad \text { Clearer, less ambiguous information/communication regarding } \\
\text { - } \quad \text { movements within worksite; } \\
\end{array}$ \\
\hline Barriers & $\begin{array}{l}\text { - } \quad \text { Time/effort required for set-up; low adaptation to context; } \\
\text { - } \quad \text { Perceived threat, particularly for more experienced workers; } \\
\text { - } \quad \text { quick on-the-spot repair); } \\
\text { - } \quad \text { Less suitable for picking up non-visual cues (e.g., tactile cues); } \\
\text { Project plans that do not accommodate learning, risk and failure with } \\
\text { innovative projects; } \\
\text { - High workload as operator needs to maintain awareness of automation } \\
\text { in early phases of adoption. }\end{array}$ \\
\hline Functional requirements & $\begin{array}{ll}\text { - } & \text { Reliable; } \\
\text { - } & \text { HMI that supports configuration; } \\
& \text { HMI that supports clear mental model of integrated nature of } \\
\text { - } & \text { Simple, robust, time-effective safety system, suitable for } \\
\text { - } & \text { Autockwork environment; } \\
\text { - } & \text { Automation able to degrade gracefully in non-routine situations; } \\
\text { - } & \text { Physical ergonomics of technology (e.g., manual handling of robots); } \\
\text { - Try to maximise adaption to context; } & \text { Replicability and reliability across multiple sites. }\end{array}$ \\
\hline $\begin{array}{l}\text { Socio-technical } \\
\text { requirements }\end{array}$ & 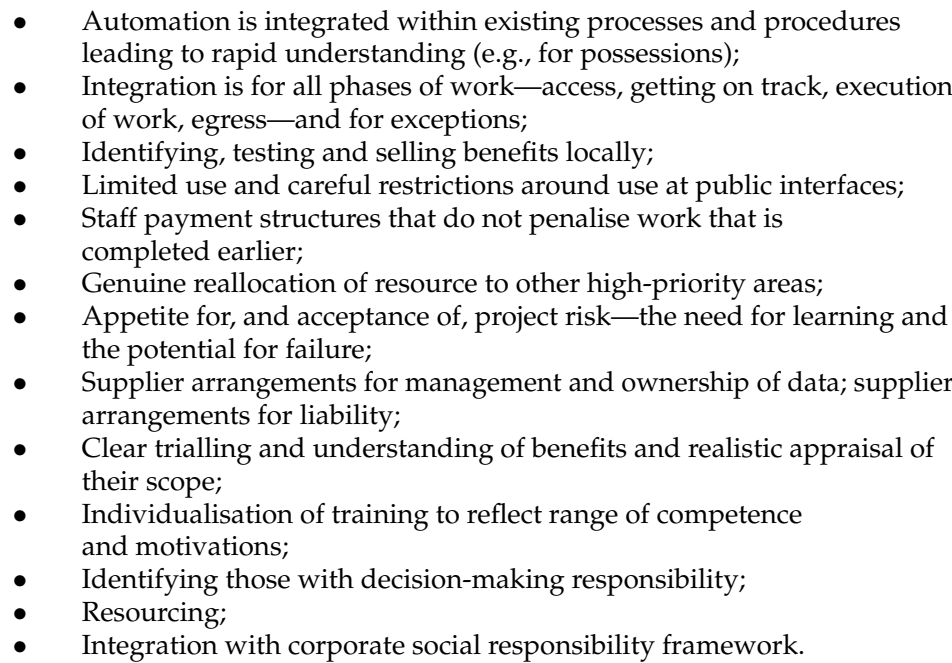 \\
\hline
\end{tabular}

Table 2. Benefits, barriers and requirements for mid-term adoption of rail infrastructure robotics.

\begin{tabular}{|c|c|}
\hline Functional description & $\begin{array}{l}\text { - One-to-few control by operator as automation becomes } \\
\text { increasingly intelligent/autonomous; } \\
\text { - } \quad \text { Increasing need to integrate with other forms of automation } \\
\text { coming online in rail (e.g., ATC, ETCS, TM); } \\
\text { - } \quad \text { Self-configuring to local conditions and context; } \\
\text { - } \quad \text { Application to an increasing range of contexts; } \\
\text { - } \quad \text { Fundamentally new forms of work and role emerging; } \\
\text { Possibility for higher levels of automation on new lines. }\end{array}$ \\
\hline
\end{tabular}


Table 2. Cont.

\begin{tabular}{|c|c|}
\hline Benefits & $\begin{array}{l}\text { A reduction in onsite presence; welfare and wellbeing } \\
\text { improvements; reduced working at remote locations; } \\
\text { Adoption of technologies which, by then, are likely to be seen } \\
\text { more routinely in the public domain; } \\
\text { - Greater autonomy of robots reduces manual handling for } \\
\text { getting to site, etc.; } \\
\text { Automatic generation to trackworking communication } \\
\text { protocols; potentially to other autonomous agents and/or } \\
\text { directly to signalling/traffic manager role; } \\
\text { - Rail staff are themselves advocates for successful automation. }\end{array}$ \\
\hline Barriers & $\begin{array}{l}\text { - High volumes of data being exacerbated by increasing volumes } \\
\text { of historical data; } \\
\text { - } \quad \text { Reduced on-track presence means loss of opportunistic } \\
\text { fault finding; } \\
\text { - Increasing autonomy leading to risks associated with underload; } \\
\text { - Complex mix of new and legacy technology. }\end{array}$ \\
\hline Functional requirements & $\begin{array}{l}\text { - Robots capable of supporting collaborative control; operator can } \\
\text { step in and out of control; } \\
\text { HMIs becoming increasing supervisory rather than for detailed } \\
\text { control of robot; } \\
\text { More advanced, intelligent proximity warning sensors and } \\
\text { safety systems to allow close integration with } \\
\text { working environment. }\end{array}$ \\
\hline $\begin{array}{l}\text { Socio-technical } \\
\text { requirements }\end{array}$ & $\begin{array}{l}\text { - Increasing introduction of infrastructure that is tailored to the } \\
\text { - } \quad \text { Inceds of automation (e.g., modularisation; standardisation); } \\
\text { - } \quad \text { Radically new procedures to exploit automation; } \\
\text { - } \quad \text { Integration skills are likely to move in-house; } \\
\text { - } \quad \text { Increasing use of robotics, etc., by the downstream supply chain; } \\
\text { - } \quad \text { Recruitment to match required skills rather than just retraining. }\end{array}$ \\
\hline
\end{tabular}

Table 3. Benefits, barriers and requirements for far-term adoption of rail infrastructure robotics.

\begin{tabular}{|c|c|}
\hline Functional description & $\begin{array}{l}\text { - One-to-many control (e.g., for fleets of UAVs); } \\
\text { - Intelligent interpretation of data; } \\
\text { - } \\
\text { - } \\
\text { Apelf-locating; able to perform multiple tasks; } \\
\text { public interfaces. }\end{array}$ \\
\hline Benefits & $\begin{array}{l}\text { - } \quad \text { Minimised working at remote locations; } \\
\text { - } \quad \text { Reduced safety exposure; } \\
\text { Benefitting from adoption of robotics, etc., from } \\
\text { other sectors; } \\
\text { - } \quad \text { Highly optimised use of railway infrastructure; } \\
\text { Increased railway performance, capacity and sustainability. }\end{array}$ \\
\hline Barriers & $\begin{array}{l}\text { - } \quad \text { Difficult to envisage from current state of the world; } \\
\text { - } \quad \text { Fundamental changes to the way the railway operates; } \\
\text { - } \quad \text { Long-term commitment and investment; } \\
\text { - } \quad \text { Perceived significant reduction in on-track staff; } \\
\text { - } \quad \text { Potential for crime from more autonomous working. }\end{array}$ \\
\hline
\end{tabular}


Table 3. Cont.

\begin{tabular}{|c|c|c|}
\hline Functional requirements & $\begin{array}{l}\bullet \\
\bullet \\
\bullet \\
\bullet\end{array}$ & $\begin{array}{l}\text { Scheduling takes place at a group level; operator controls } \\
\text { the schedule; } \\
\text { Schedule is optimised for factors such as workload; } \\
\text { Multisensory HMI; } \\
\text { Potential for direct comms link with many rail systems (e.g., } \\
\text { robots with direct access to real-time timetable). }\end{array}$ \\
\hline Socio-technical requirements & & $\begin{array}{l}\text { Significant organisational change to take advantage } \\
\text { of automation; } \\
\text { Understanding of integration with other forms of } \\
\text { automation that are likely to be present; } \\
\text { Consideration of crime likely to emerge from more } \\
\text { autonomous equipment. }\end{array}$ \\
\hline
\end{tabular}

There are also some more specific recommendations below.

\subsubsection{Ontology}

Part of successfully determining the characteristics of automation will be to define a structure or ontology that expresses the important features to consider when developing user-centred automation for rail maintenance. While many of these may be obvious to those in a rail maintenance role, they may be less obvious to third-party designers or integrators of automation. This includes:

- $\quad$ The stages of work (planning, access, performing the work, egress).

- Dimensions of safety (safety of the robot, safe work quality, safety of workers, safe action in unexpected events). The important point to note here is that safety arrangements are likely to be more complex than those previously encountered in many, more 'closed', automation working environments (e.g., manufacturing, warehousing) and therefore needs both careful planning and appropriate reflection in standards, training, working practices and the design of the behaviours of the automation.

- Types of factors-user, team, organisational and societal.

- Maturity levels of the technology.

- Relevant rail standards.

\subsubsection{Structured Engagement}

The interviews highlighted the importance of structured engagement with all stakeholders. This includes members of the public who may be affected by, or will be in proximity to, automation, as well as staff at different levels of the organisation. This must be a continuous activity across the life of any robotic deployment project-not just 'evaluation' towards the end.

An explicit, prolonged and managed programme of culture change is essential to encourage support. Automation needs to be part of the culture of maintenance, as does a data-led approach. This requires leadership from senior management and clear communication of benefits to encourage the culture change. The frameworks proposed by [22] or [53] would make an ideal starting point and should be adapted for the rail context.

\subsubsection{Procedural Design}

Procedural design involves the approach of embedding automation in working practice. Critically, this is not a user guide of how to use automation but more how to achieve work in a manner that also describes the role and application of automation. For the time being, it might be beneficial to base this procedural design around existing procedures (e.g., the use of other forms of plant). Ref. [52] provided an initial framework that can be adapted to robotic work. 


\subsection{Limitations}

There are a number of limitations of the study. Firstly, while few participants were able to give a perspective across all major themes, and across both short and long term, the combination of expertise from within and beyond rail gave coverage across all areas. In addition, participants from the rail sector tended to focus more on the short-term implications of automation typically because they had in-depth understanding of current procedures and near-term planned changes. Non-rail participants were able to comment on issues relevant to the long-term, either because they came from sectors where robotics was more mature (e.g., manufacturing) or because they were less influenced by any prior experience of rail maintenance processes. Nonetheless, a tuning of the method (e.g., through the use of video scenarios) might help with visualising future robotics concepts to all participant groups.

Secondly, while this work looked at societal issues and public engagement, it did not as yet directly involve the public. A next phase of work could engage in focus groups or surveys to directly understand public perceptions of robotics.

Thirdly, this work took a UK perspective on rail robotics. The findings in this paper should be tested in other countries that might vary in terms of the operation of their railways [73], attitudes to rail innovation [74], safety culture [75] and national culture [59].

\section{Conclusions}

Railways are a key sustainable transportation mode, and the long-term health and viability of the railways are vital to providing low-carbon transportation. Robotics have an important role to play in the safe, effective maintenance of the railways. The design and deployment of robotics must be approached in a manner that takes into account the needs of users, teams of people working with and around the robot, organisational factors and societal factors. This paper used a qualitative approach to identify the human and organisational challenges for rail infrastructure robotics. While the results point to many of the expected issues of robotics (trust, acceptance, business change), a number of issues were identified that were specific to rail. Examples include the importance of considering the whole maintenance task lifecycle, conceptualizing robotic teamworking within the structures of rail maintenance worksites, the complex upstream (robotics suppliers) and downstream (third-party maintenance contractors) supply chain implications of robotic deployment and the public acceptance of robotics in an environment that often comes into direct contact with passenger and people around the railways. The practical contribution of this work is a number of recommendations both in terms of functional requirements and socio-technical requirements for acceptance and effectiveness of rail infrastructure robotics. The scientific benefit is an evidence-based framework to understand the range of human and organisational factors that can be used to underpin future research in the functional design of robotics, human factors work in robotics (e.g., in robotic HMI) and in organisational change.

Author Contributions: Conceptualisation, D.G., J.C.-P. and N.D.; Formal analysis, D.G., N.D. and S.J.; Investigation, D.G., N.D. and S.J.; Methodology, D.G. and A.H.; Resources, J.C.-P. and A.H.; Validation, B.R.; Writing - original draft, D.G.; Writing—review and editing, J.C.-P., N.D., B.R. and A.H. All authors have read and agreed to the published version of the manuscript.

Funding: This work was funded by Network Rail.

Institutional Review Board Statement: This study design and associated materials were reviewed and approved by the Faculty of Engineering Ethics Committee, University of Nottingham, UK, June 2017.

Informed Consent Statement: Informed consent was obtained from all the participants involved in the study.

Acknowledgments: The authors are grateful for the time and input of all the subject matter experts involved in this work. 
Conflicts of Interest: The authors declare no conflict of interest.

\section{References}

1. Railway Accident Investigation Branch (RAIB). Two Incidents Involving Track Workers between Clapham Junction and Earlsfield, 8 March 2011, Department for Transport. 2012. Available online: https:/ /www.gov.uk/raib-reports/two-incidents-involvingtrack-workers-between-clapham-junction-and-earlsfield (accessed on 5 January 2022).

2. Railway Accident Investigation Branch (RAIB). Track Workers Struck by a Train at Margam, 3 July 2019, Department for Transport. 2020. Available online: https://www.gov.uk/raib-reports/report-11-2020-track-workers-struck-by-a-train-at-margam (accessed on 5 January 2022).

3. Farrington-Darby, T.; Pickup, L.; Wilson, J.R. Safety culture in railway maintenance. Saf. Sci. 2005, 43, 39-60. [CrossRef]

4. Wilson, J.R.; Ryan, B.; Schock, A.; Ferreira, P.; Smith, S.; Pitsopoulos, J. Understanding safety and production risks in rail engineering planning and protection. Ergonomics 2009, 52, 774-790. [CrossRef]

5. Golightly, D.; Ryan, B.; Dadashi, N.; Pickup, L.; Wilson, J. Use of scenarios and function analyses to understand the impact of situation awareness on safe and effective work on rail tracks. Saf. Sci. 2013, 56, 52-62. [CrossRef]

6. Luva, B.; Naweed, A. Authority gradients between team workers in the rail environment: A critical research gap. Theor. Issues Ergon. Sci. 2021, 1-29. [CrossRef]

7. Riley, D. Manual Handling in the Rail Sector in South Wales. HSL/2006/53. Available online: https://www.hse.gov.uk/research/ hsl_pdf/2006/hs10653.pdf (accessed on 5 January 2022).

8. Salguero-Caparrós, F.; Martínez-Rojas, M.; Pardo-Ferreira, M.D.C.; Rubio-Romero, J.C. Integration of Ergonomics in the Study of Catenary Execution Projects. In Modern Trends and Research in Intermodal Transportation; Springer: Cham, Switzerland, 2019; pp. 503-511.

9. National Skills Academy for Rail (2020) Back on Track: Gearing up to Meet the Increased Demand for Talent in the Rail Industry. Available online: https://www.cityandguildsgroup.com/-/media/cgg-website/documents/cgg-back-on-track-report (accessed on 5 January 2022).

10. Dadashi, N.; Wilson, J.R.; Golightly, D.; Sharples, S. A framework to support human factors of automation in railway intelligent infrastructure. Ergonomics 2014, 57, 387-402. [CrossRef] [PubMed]

11. Montero, R.; Victores, J.; Martínez, S.; Jardón, A.; Balaguer, C. Past, present and future of robotic tunnel inspection. Autom. Constr. 2015, 59, 99-112. [CrossRef]

12. Vithanage, R.K.W.; Harrison, C.S.; DeSilva, A.K.M. Importance and Applications of Robotic and Autonomous Systems (RAS) in Railway Maintenance Sector: A Review. Computers 2019, 8, 56. [CrossRef]

13. Rahman, M.; Liu, H.; Cardenas, I.D.; Starr, A.; Hall, A.; Anderson, R. Towards an Autonomous RIRS: Design, Structure Investigation and Framework. In Proceedings of the 2021 7th International Conference on Mechatronics and Robotics Engineering (ICMRE), Budapest, Hungary, 3-5 February 2021; pp. 164-168.

14. Rahimi, M.; Liu, H.; Rahman, M.; Carcel, C.R.; Durazo-Cardenas, I.; Starr, A.; Anderson, R. Localisation and Navigation Framework for Autonomous Railway Robotic Inspection and Repair System. In Proceedings of the 10th International Conference on Through-life Engineering Service, Enschede, The Netherlands, 16-17 November 2021.

15. Durazo-Cardenas, I.; Starr, A.; Turner, C.J.; Tiwari, A.; Kirkwood, L.; Bevilacqua, M.; Tsourdos, A.; Shehab, E.; Baguley, P.; $\mathrm{Xu}, \mathrm{Y}$; et al. An autonomous system for maintenance scheduling data-rich complex infrastructure: Fusing the railways' condition, planning and cost. Transp. Res. Part C Emerg. Technol. 2018, 89, 234-253. [CrossRef]

16. Bertrand, S.; Raballand, N.; Viguier, F.; Muller, F. Ground risk assessment for long-range inspection missions of railways by UAVs. In Proceedings of the 2017 International Conference on Unmanned Aircraft Systems (ICUAS), Athens, Greece, 13-16 June, 2017; pp. 1343-1351.

17. IEA and UIC (2012) Railway Handbook 2012-Energy Consumption and $\mathrm{CO}_{2}$ Emissions, International Energy Agency. Available online: http:/ / www.uic.org/IMG/pdf/iea-uic_energy_consumption_and_co2_emission_of_world_railway_sector.pdf (accessed on 20 September 2019).

18. Van Loon, R.; Rietveld, P.; Brons, M. Travel-time reliability impacts on railway passenger demand: A revealed preference analysis. J. Transp. Geogr. 2011, 19, 917-925. [CrossRef]

19. Aboelmaged, M. Predicting e-readiness at firm-level: An analysis of technological, organizational and environmental (TOE) effects on e-maintenance readiness in manufacturing firms. Int. J. Inf. Manag. 2014, 34, 639-651. [CrossRef]

20. Jonsson, K.; Holmström, J.; Levén, P. Organizational dimensions of e-maintenance: A multi-contextual perspective. Int. J. Syst. Assur. Eng. Manag. 2010, 1, 210-218. [CrossRef]

21. Charalambous, G.; Fletcher, S.; Webb, P. Identifying the key organisational human factors for introducing human-robot collaboration in industry: An exploratory study. Int. J. Adv. Manuf. Technol. 2015, 81, 2143-2155. [CrossRef]

22. Charalambous, G.; Fletcher, S.R.; Webb, P. The development of a Human Factors Readiness Level tool for implementing industrial human-robot collaboration. Int. J. Adv. Manuf. Technol. 2017, 91, 2465-2475. [CrossRef]

23. Winfield, A.F.T.; Jirotka, M. Ethical governance is essential to building trust in robotics and artificial intelligence systems. Philos. Trans. R. Soc. London. Ser. A Math. Phys. Eng. Sci. 2018, 376, 20180085. [CrossRef]

24. Read, G.J.; Salmon, P.M.; Lenné, M.G. Sounding the warning bells: The need for a systems approach to understanding behaviour at rail level crossings. Appl. Ergon. 2013, 44, 764-774. [CrossRef] [PubMed] 
25. Bainbridge, L. Ironies of automation. Automatica 1983, 19, 775-779. [CrossRef]

26. Papadimitriou, E.; Schneider, C.; Tello, J.A.; Damen, W.; Vrouenraets, M.L.; Broeke, A.T. Transport safety and human factors in the era of automation: What can transport modes learn from each other? Accid. Anal. Prev. 2020, 144, 105656. [CrossRef]

27. Hollnagel, E.; Woods, D.D. Joint Cognitive Systems: Foundations of Cognitive Systems Engineering; CRC Press: Boca Raton, FL, USA, 2005

28. Hoffman, R.R.; Woods, D.D. Beyond Simon's slice: Five fundamental trade-offs that bound the performance of macrocognitive work systems. IEEE Intell. Syst. 2011, 26, 67-71. [CrossRef]

29. Young, T.; Naweed, A. The Impact of Automatic Train Protection on Ways of Working in Australian Rail: Preliminary Findings from the Driver Perspective. In Proceedings of the Ergonomics and Human Factors 2017 Conference, Daventry, UK, 25-27 April 2017.

30. National Research Council. Human-System Integration in the System Development Process: A New Look; National Academies Press: Washington, DC, USA, 2007.

31. Wilson, J.R. Fundamentals of systems ergonomics/human factors. Appl. Ergon. 2014, 45, 5-13. [CrossRef]

32. Wilson, J.R.; Sharples, S. Methods in the Understanding of Human Factors. In Evaluation of Human Work; CRC Press: Boca Raton, FL, USA, 2015; pp. 34-69.

33. Karsh, B.-T.; Holden, R.J.; Alper, S.J.; Or, C.K.L. A human factors engineering paradigm for patient safety: Designing to support the performance of the healthcare professional: Figure 1. Qual. Saf. Health Care 2006, 15, i59-i65. [CrossRef]

34. Golightly, D.; Kefalidou, G.; Sharples, S. A cross-sector analysis of human and organisational factors in the deployment of data-driven predictive maintenance. Inf. Syst. E-Bus. Manag. 2017, 16, 627-648. [CrossRef]

35. Meissner, A.; Trübswetter, A.; Conti-Kufner, A.S.; Schmidtler, J. Friend or Foe? Understanding Assembly Workers' Acceptance of Human-robot Collaboration. ACM Trans. Hum. Robot Interact. 2021, 10, 1-30. [CrossRef]

36. Hancock, P.A.; Billings, D.R.; Schaefer, K.E. Can you trust your robot? Ergon. Des. 2011, 19, 24-29. [CrossRef]

37. Ososky, S.; Schuster, D.; Phillips, E.; Jentsch, F.G. Building Appropriate Trust in Human-Robot Teams. In Proceedings of the AAAI Spring Symposium: Trust and Autonomous Systems, Wednesday. Stanford, CA, USA, 24-26 March 2003.

38. Balfe, N.; Wilson, J.R.; Sharples, S.; Clarke, T. Development of design principles for automated systems in transport control. Ergonomics 2011, 55, 37-54. [CrossRef]

39. Dixon, S.R.; Wickens, C.D. Automation Reliability in Unmanned Aerial Vehicle Control: A Reliance-Compliance Model of Automation Dependence in High Workload. Hum. Factors J. Hum. Factors Ergon. Soc. 2006, 48, 474-486. [CrossRef]

40. Charalambous, G.; Fletcher, S.R. Trust in Industrial Human-Robot Collaboration. In 21st Century Industrial Robot: When Tools Become Collaborators; Springer; Cham, Switzerland, 2022; pp. 87-103. [CrossRef]

41. Sanders, T.L.; Volante, W.; Stowers, K.; Kessler, T.; Gabracht, K.; Harpold, B.; Oppold, P.; Hancock, P.A. The Influence of Robot Form on Trust. In Proceedings of the 59th Annual Meeting of the Human Factors and Ergonomics Society, Garden Grove, CA, USA, 6-8 June 2016.

42. Charalambous, G.; Fletcher, S.; Webb, P. The Development of a Scale to Evaluate Trust in Industrial Human-robot Collaboration. Int. J. Soc. Robot. 2016, 8, 193-209. [CrossRef]

43. Klein, G.; Woods, D.D.; Bradshaw, J.; Hoffman, R.R.; Feltovich, P.J. Ten Challenges for Making Automation a "Team Player" in Joint Human-Agent Activity. IEEE Intell. Syst. 2004, 19, 91-95. [CrossRef]

44. Mouloua, M.; Gilson, R.; Kring, J.; Hancock, P. Workload, Situation Awareness, and Teaming Issues for UAV/UCAV Operations. Proc. Hum. Factors Ergon. Soc. Annu. Meet. 2001, 45, 162-165. [CrossRef]

45. Cummings, M.; Mitchell, P. Predicting Controller Capacity in Supervisory Control of Multiple UAVs. IEEE Trans. Syst. Man Cybern. Part A Syst. Hum. 2008, 38, 451-460. [CrossRef]

46. Cummings, M.L.; Nehme, C.E. Modeling the Impact of Workload in Network Centric Supervisory Control Settings. In Proceedings of the 2nd Annual Sustaining Performance under Stress Symposium, College Park, MD, USA, 25 February 2009.

47. Scholtz, J. Theory and evaluation of human robot interactions. In Proceedings of the 36th Annual Hawaii International Conference on System Sciences, Big Island, HI, USA, 3-6 January 2003; p. 10.

48. Woods, D.D.; Branlat, M. Hollnagel's test: Being 'in control'of highly interdependent multi-layered networked systems. Cogn. Technol. Work 2010, 12, 95-101. [CrossRef]

49. Schock, A.; Ryan, B.; Wilson, J.; Clarke, T.; Sharples, S. Visual scenario analysis: Understanding human factors of planning in rail engineering. Prod. Plan. Control 2010, 21, 386-398. [CrossRef]

50. Drury, J.L.; Riek, L.; Rackliffe, N. A decomposition of UAV-related situation awareness. In Proceedings of the 1st ACM SIGCHI/SIGART Conference on Human-Robot Interaction-HRI '06, Salt Lake City, UT, USA, 2-3 March 2006; pp. 88-94.

51. Ciocoiu, L.; Siemieniuch, C.E.; Hubbard, E.M. The changes from preventative to predictive maintenance: The organisational challenge. In Proceedings of the 5th International Rail Human Factors Conference, London, UK, 14-17 September 2015.

52. Ryan, B.; Golightly, D.; Pickup, L.; Reinartz, S.; Atkinson, S.; Dadashi, N. Human functions in safety-Developing a framework of goals, human functions and safety relevant activities for railway socio-technical systems. Saf. Sci. 2021, 140, 105279. [CrossRef]

53. Kopp, T.; Baumgartner, M.; Kinkel, S. Success factors for introducing industrial human-robot interaction in practice: An empirically driven framework. Int. J. Adv. Manuf. Technol. 2021, 112, 685-704. [CrossRef] 
54. Goldberg, K.; Kehoe, B. Cloud Robotics and Automation: A Survey of Related Work. EECS Department, University of California, Berkeley, Tech. Rep. UCB/EECS-2013-5. Available online: https://static.aminer.cn/upload/pdf/1658/787/1637/5eb3a9e59e795 e177f12a3d6_0.pdf (accessed on 5 January 2022).

55. Charalambous, G.; Stout, M. Optimising train axle inspection with the implementation of human-robot collaboration: A human factors perspective. In Proceedings of the 2016 IEEE International Conference on Intelligent Rail Transportation (ICIRT), Birmingham, UK, 23-25 August 2016; pp. 254-258.

56. Lou, E.; Goulding, J. The pervasiveness of e-readiness in the global built environment arena. J. Syst. Inf. Technol. 2010, 12, 180-195. [CrossRef]

57. Baines, T.; Lightfoot, W.H. Servitization of the manufacturing firm: Exploring the operations practices and technologies that deliver advanced services. Int. J. Oper. Prod. Manag. 2013, 34, 2-35. [CrossRef]

58. Kefalidou, G.; Golightly, D.; Sharples, S. Identifying rail asset maintenance processes: A human-centric and sensemaking approach. Cogn. Technol. Work 2018, 20, 73-92. [CrossRef]

59. Hofstede, G.; Hofstede, G.J.; Minkov, M. Cultures and Organizations: Software of the Mind, 3rd ed.; McGraw-Hill: New York, NY, USA, 2010.

60. Rice, S.; Kraemer, K.; Winter, S.; Mehta, R.; Dunbar, V.; Rosser, T.; Moore, J. Passengers from India and the United States Have Differential Opinions about Autonomous Auto-Pilots for Commercial Flights. Int. J. Aviat. Aeronaut. Aerosp. 2014, 1, 3. [CrossRef]

61. Evers, V.; Maldonado, H.; Brodecki, T.; Hinds, P. Relational vs. group self-construal: Untangling the role of national culture in HRI. In Human-Robot Interaction (HRI). In Proceedings of the 2008 3rd ACM/IEEE International Conference, Amsterdam, The Netherlands, 12-15 March 2008; pp. 255-262.

62. Ezer, N.; Fisk, A.D.; Rogers, W.A. Attitudinal and Intentional Acceptance of Domestic Robots by Younger and Older Adults. In International Conference on Universal Access in Human-Computer Interaction; Springer: Berlin/Heidelberg, Germany, 2009; pp. 39-48.

63. Cardon, P.W.; Marshall, B.A. National culture and technology acceptance: The impact of uncertainty avoidance. Issues Inf. Syst. 2008, 9, 103-110.

64. Rau, P.P.; Li, Y.; Li, D. Effects of communication style and culture on ability to accept recommendations from robots. Comput. Hum. Behav. 2009, 25, 587-595. [CrossRef]

65. Bruno, B.; Chong, N.Y.; Kamide, H.; Kanoria, S.; Lee, J.; Lim, Y.; Pandey, A.K.; Papadopoulos, C.; Papadopoulos, I.; Pecora, F.; et al. Paving the way for culturally competent robots: A position paper. In Proceedings of the 2017 26th IEEE International Symposium on Robot and Human Interactive Communication (RO-MAN), Lisbon, Portugal, 29-31 August 2017; pp. 553-560.

66. Visioncritical (2014) Drone Awareness and Perceptions: A 3 Country Study. Available online: http:/ / odesi1.scholarsportal.info/ documentation/drones-survey/2014/Drone_Awareness_and_Perceptions.pdf (accessed on 5 January 2022).

67. Ryan, B.; Kallberg, V.-P.; Rådbo, H.; Havârneanu, G.M.; Silla, A.; Lukaschek, K.; Burkhardt, J.-M.; Bruyelle, J.-L.; El-Koursi, E.-M.; Beurskens, E.; et al. Collecting evidence from distributed sources to evaluate railway suicide and trespass prevention measures. Ergonomics 2018, 61, 1433-1453. [CrossRef]

68. Silla, A.; Luoma, J. Opinions on railway trespassing of people living close to a railway line. Saf. Sci. 2012, 50, 62-67. [CrossRef]

69. Sturges, J.E.; Hanrahan, K.J. Comparing Telephone and Face-to-Face Qualitative Interviewing: A Research Note. Qual. Res. 2004, 4, 107-118. [CrossRef]

70. Hignett, S.; McDermott, H. Qualitative methodology. In Evaluation of Human Work, 4th ed.; Wilson, J.R., Sharples, S., Eds.; CRC Press: Boca Raton, FL, USA, 2015; pp. 119-138.

71. Office of Road and Rail (2021) Railways and Other Guided Transport Systems (Safety) Regulations 2006 (ROGS). Available online: https://www.orr.gov.uk/guidance-compliance/rail/health-safety/laws/rogs (accessed on 5 January 2022).

72. Stiles, S.; Ryan, B.; Golightly, D. Evaluating attitudes to safety leadership within rail construction projects. Saf. Sci. 2018, 110, 134-144. [CrossRef]

73. Schipper, D.; Gerrits, L. Differences and similarities in European railway disruption management practices. J. Rail Transp. Plan. Manag. 2018, 8, 42-55. [CrossRef]

74. Hahne, E.; Ryan, B.; Smith, S. Safety First! Cultural Differences in the Use of Track Warning Systems. In Proceedings of the Ergonomics and Human Factors Conference, Daventry, UK, 25-27 April 2017.

75. Noort, M.C.; Reader, T.W.; Shorrock, S.T.; Kirwan, B. The relationship between national culture and safety culture: Implications for international safety culture assessments. J. Occup. Organ. Psychol. 2016, 89, 515-538. [CrossRef] 